\title{
Letter \\ Single-Stage Rotation-Decoupled Detector for Oriented Object
}

\author{
Bo Zhong ${ }^{1,2}$ and Kai Ao ${ }^{1,2, * \mathbb{C}}$ \\ 1 College of Computer Science and Technology, University of Posts and Telecommunications, \\ Chongqing 400065, China; zhongbo@radi.ac.cn \\ 2 State Key Laboratory of Remote Sensing Science, Aerospace information Research Institute, \\ Chinese Academy of Sciences, Beijing 100101, China \\ * Correspondence: s180201046@stu.cqupt.edu.cn
}

Received: 1 September 2020; Accepted: 1 October 2020; Published: 8 October 2020

\begin{abstract}
Oriented object detection has received extensive attention in recent years, especially for the task of detecting targets in aerial imagery. Traditional detectors locate objects by horizontal bounding boxes (HBBs), which may cause inaccuracies when detecting objects with arbitrary oriented angles, dense distribution and a large aspect ratio. Oriented bounding boxes (OBBs), which add different rotation angles to the horizontal bounding boxes, can better deal with the above problems. New problems arise with the introduction of oriented bounding boxes for rotation detectors, such as an increase in the number of anchors and the sensitivity of the intersection over union (IoU) to changes of angle. To overcome these shortcomings while taking advantage of the oriented bounding boxes, we propose a novel rotation detector which redesigns the matching strategy between oriented anchors and ground truth boxes. The main idea of the new strategy is to decouple the rotating bounding box into a horizontal bounding box during matching, thereby reducing the instability of the angle to the matching process. Extensive experiments on public remote sensing datasets including DOTA, HRSC2016 and UCAS-AOD demonstrate that the proposed approach achieves state-of-the-art detection accuracy with higher efficiency.
\end{abstract}

Keywords: object detection; aerial images; arbitrary-oriented; convolutional neural network

\section{Introduction}

With the increasing number of applications based on convolutional neural networks (CNNs) in the field of computer vision, object detection algorithms have been developed rapidly. Existing detectors [1-4] have achieved promising results on real-life datasets including MS COCO [5] and VOC2007 [6]. Related models typically use horizontal bounding boxes (HBBs) to locate targets. Most targets in remote sensing imageries are characterized by an arbitrary directionality, high aspect ratio and dense distribution; consequently, the models based on HBBs may cause serious overlap and noise. Subsequently, the rotating bounding box was devised to deal with these targets, with the advantages of capturing the target with better accuracy and introducing the least background noise. In addition, oriented bounding boxes (OBBs) separate densely distributed targets perfectly and thus avoid the overlapping of the adjacent bounding boxes. Specifically, for the detection of ships and vehicles, oriented detectors [7-11] based on rotating bounding boxes perform well.

However, with the introduction of the rotating bounding box, due to the sensitivity of the intersection over union (IoU) to changes in angle, some problems also arise. A small angle change will cause a rapid drop in the IoU, which will lead to inaccurate detection. The usage of oriented anchors will lead to a sharp increase in the number of anchors. As a result of these problems, the IoU between the matched oriented anchor and the ground truth box fluctuates dramatically with the change of 
the angle between the principal axes, especially when the aspect ratio of the bounding box is large. Recent studies [12-18] have proposed a series of optimization schemes for oriented object detection, but there is still much room for improvement in this area of research.

In this paper, we propose a novel single-stage detector for effective and accurate oriented object detection. Specifically, we present a new rotation-decoupled anchor matching strategy, which considers $\mathrm{OBB}$ as a combination of the HBB and angle. The new matching process is based on the IoU of the rotation-decoupled bounding box instead of the OBB. The proposed strategy does not cause regression parameters to change, and it can play a positive role in the learning of the detector. Firstly, the HBB exhibits better stability during the matching process than the OBB. Secondly, the proposed strategy only introduces a target variable based on the HBB. Compared with other rotation detectors that need to add extra anchors with different angles, the new method requires fewer anchors, and the amount of calculation is therefore reduced. In order to adapt to the detection frame of the HBB, we redesigned the representation method of the OBB. The newly designed representation method is not affected by angle changes when calculating the IoU between anchors and ground truth boxes. The new method classifies the OBBs according to the spindle direction into two categories, which naturally correspond to the HBBs in turn. The proposed strategy does not involve changes in the model structure, so it can be easily applied to various detection models with different structures. Based on the above ideas, we have implemented the rotation-decoupled detector (RDD). The proposed approach achieves state-of-the-art performance with high efficiency on three public remote sensing datasets annotated with OBBs: DOTA [19], HRSC2016 [20] and UCAS-AOD [21].

In summary, the main contributions of this paper are threefold: (1) we propose a novel anchor matching strategy for OBBs; (2) we adjust the implementation of the new strategy so that it can be easily embedded in many mainstream detectors without increasing the amount of calculation; and (3) based on the proposed anchor matching strategy, we construct a new rotation detector without introducing complex network structures. The proposed model is highly efficient and achieves state-of-the-art accuracy on multiple detection benchmarks with multi-oriented objects.

\section{Related Work}

\subsection{Horizontal Object Detection}

Object detection algorithms typically use horizontal bounding boxes to locate targets. At the beginning of the application of the convolutional neural network (CNN) to the object detection task, $\mathrm{R}-\mathrm{CNN}$ [22] uses a selective search algorithm to generate category-independent region proposals and then extracts fixed-length feature vectors from each region proposal through $\mathrm{CNN}$ for classification. Due to the success of R-CNN, many models have been developed based on it. Fast R-CNN [23] introduces RoI Pooling to process region proposals, which effectively reduces computational redundancy. Faster R-CNN [24] uses a region proposal network (RPN) to generate region proposals so that the model can be trained end-to-end. At this point, the structure of the two-stage detector is basically determined: generating region proposals and then predicting the precise location of the targets and the corresponding category labels. According to the characteristics of the two-stage detector, Mask R-CNN [25] embeds the image segmentation task into the detector's second-stage task, effectively improving the accuracy of instance segmentation. In order to achieve real-time detection, single-stage detectors have appeared that perform two stages simultaneously. YOLO [26] grids the images and performs simultaneous category prediction and position regression directly on the feature map output from CNN. SSD [1] makes full use of multiple feature maps with different resolutions to naturally predict targets of different sizes. In order to solve the category imbalance problem of single-stage detectors, RetinaNet [2] proposes Focal Loss, which is a dynamically scaled cross entropy loss. RefineDet [3] uses the anchor refinement module (ARM) and the object detection module $(\mathrm{ODM})$ to imitate the two-stage structure and produces accurate detection results with high efficiency. EfficientDet [4] realizes easy and fast multi-scale feature fusion through a weighted bi-directional 
feature pyramid network (BiFPN). EfficientDet [4] also proposes a new compound scaling method to make the model complexity and accuracy adjustable. The above-mentioned methods are all anchor-based; in recent years, anchor-free methods have begun to emerge. CornerNet [27] locates the target by learning a pair of key points: the top-left corner and the bottom-right corner. CenterNet [28] models the object as a key point and directly predicts the center point and other properties of the object. FCOS [29] further optimizes the performance of anchor-free detectors and unifies the detection process with other fully convolutional network (FCN)-solvable tasks. In general, the two-stage detector maintains a high detection accuracy rate, while the single-stage detector achieves a balance between efficiency and accuracy.

\subsection{Oriented Object Detection}

The application of rotating object detection in the field of oriented object detection in aerial imagery is being extensively studied. The anchor-based method shows strong stability for multiple multi-oriented object detection benchmarks. Considering the difficulty of the anchor-based method for high aspect ratio object detection, the anchor-free method is also widely applied. For remote sensing object detection, RoI Transformer [13] learns the transformation from HRoIs to RRoIs and then extracts the rotation-invariant features from the RRoI through rotated position-sensitive RoI alignment. $\mathrm{R}^{3}$ Det [30] uses a combination strategy, first performing rapid detection based on horizontal anchor boxes and then performing oriented object detection based on refined rotating anchor boxes. In [16], a novel method of rotating bounding box representation based on a gliding vertex on the horizontal bounding box is introduced to describe multi-oriented objects more accurately and avoid confusion issues. Considering the background noise interference caused by the horizontal bounding box, SCRDet++ [31] proposes instance level denoising (InLD) for small and cluttered objects. The anchor-free methods also show strong competitiveness in remote sensing object detection. DHN [17] presents a dynamic refinement network which alleviates the misalignment between receptive fields and objects by a feature selection module (FSM) and refines the prediction in an object-wise manner by using a dynamic refinement head (DRH). Regarding the angular periodicity problem in rotating object detection, APE [18] represents the angle as continuously changing periodic vectors to avoid ambiguity. In addition, APE [18] designs a length-independent IoU (LIIoU) for long objects to make the detector more robust.

\section{Proposed Method}

The proposed RDD is designed based on an FPN [32] architecture which uses multi-scale feature maps for detection and is currently widely adopted. The structure of RDD is simple compared to many current models. In addition, as a rotation detector, the learning of position parameters is also more concise. The arbitrarily oriented targets are represented as rotated bounding boxes which are more accurate than the horizontal boundary frame. However, the IoU is sensitive to changes of angle. We found in experiments that, by designing appropriate learning targets, even without using a rotating anchor box, the angle can be learned accurately. Specifically, we designed a new rotated bounding box representation method for this. Furthermore, a new rotation-decoupled anchor matching strategy is proposed to optimize the learning of the position information of the arbitrary oriented target. The positive and negative sample balance strategy is adopted to deal with foreground-background class imbalance. The experimental results show that the proposed method achieves state-of-the-art accuracy on both single-category and multi-category rotation detection datasets.

\subsection{Network Architecture}

RDD has a lightweight network architecture, which is illustrated in Figure 1. The type of each operation is shown at the bottom of Figure 1. At first, the multi-scale feature maps were obtained from the backbone network, and the widely used ResNet101 [33] was chosen for experiments in this paper. Secondly, the multi-scale feature maps were inputted into the pyramid structure network for feature 
fusion; the details of the pyramid structure network are illustrated in Figure 1. The pyramid network realizes the transmission of semantic information, which is helpful for multi-scale object detection. In order to connect the feature layers of different scales, we up-sampled the feature map and summed it with the feature map of the previous layer in an element-wise manner. We added a convolutional layer before and after the summation to ensure the discriminability of features for detection. Finally, the prediction layers output the classification and regression. Classification and regression use two prediction layers with the same structure, and they only differ in the number of output channels. For classification, the number of output channels is $a \times c$; for regression, the number of output channels is $a \times 5 . a$ and $c$ refer to the number of anchors and the number of categories. The illustrated structure can also be extended to more layers in practice. In this study, a rotation-decoupled anchor matching strategy was designed at the training stage, and only horizontal anchors were subsequently employed by the proposed model instead of the oriented anchors.

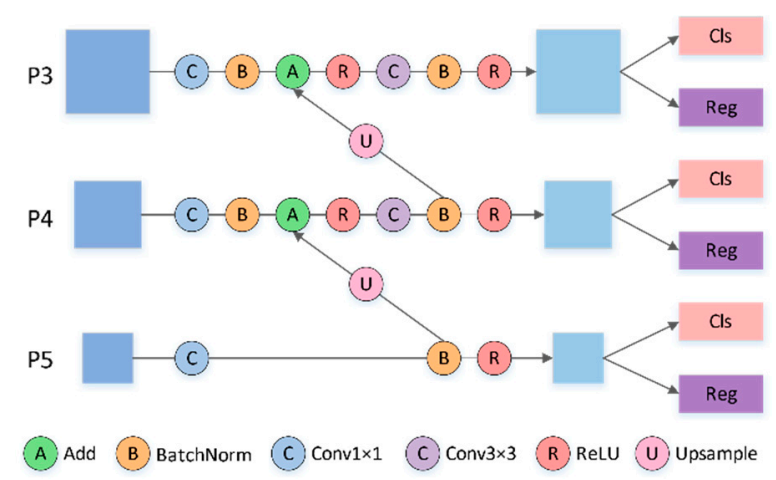

Figure 1. Architecture of the proposed rotation-decoupled detector. The darker blue squares represent the feature maps with different scales from the backbone network, the lighter blue squares are the feature maps with different scales after fusion, the circle represents an operation, and the arrows indicate the order of operations.

\subsection{Rotated Bounding Box Representation}

The HBB has good robustness but insufficient accuracy. It is usually represented by $(x, y, w, h)$, where $(x, y)$ is the center and $w$ and $h$ are the lengths of the bounding box along the $X$ and $Y$ axes, respectively. The OBB is more accurate but less robust. It is usually represented by $(x, y, h, w, \theta)$, where $\theta$ is the angle of the bounding box; however, the periodicity of $\theta$ usually causes a sudden IoU drop, and inaccurate object detection is subsequently avoidable, especially in case of large aspect ratios. Both the HBB and OBB have different advantages and disadvantages, respectively; therefore, the advantages of the $\mathrm{HBB}$ and $\mathrm{OBB}$ representation methods are combined to redefine the new bounding box.

Traditionally, an HBB defined as $(x, y, w, h)$ is different to an HBB defined as $(x, y, h, w)$; however, they are redefined as $\mathrm{HBB}_{\mathrm{h}}$ as the $\mathrm{HBB}$ in the horizontal direction and $\mathrm{HBB}_{\mathrm{v}}$ as the same $\mathrm{HBB}$ in the vertical direction. Therefore, for any OBB, we can find a corresponding $\mathrm{HBB}_{\mathrm{h} / \mathrm{v}}$. They have the same shape and center point, and the angle $\theta$ between them is within $[-\pi / 4, \pi / 4]$. We redefine $\theta$ as the angle of the new bounding box. Figure 2 shows the redefinition of the bounding box intuitively. Through the new definition of the bounding box, the $\mathrm{HBB}_{\mathrm{h} / \mathrm{v}}$ is used as the ground truth box to match with the anchors, and it effectively avoids the problem of angle periodicity induced by the OBB. 

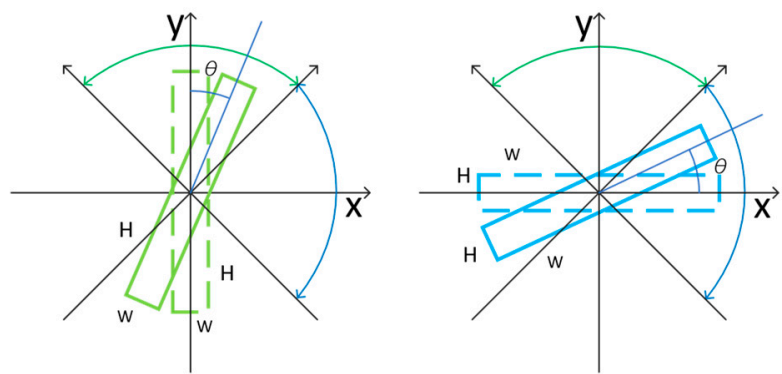

Figure 2. Redefinition of the bounding box to decouple an oriented bounding box (OBB) as a horizontal bounding box $\left(\mathrm{HBB}_{\mathrm{h} / \mathrm{v}}\right)$ and the acute angle between $\mathrm{HBB}$ and OBB. The dashed boxes represent the HBB corresponding to the OBB (the solid boxes).

\subsection{Anchor Setting}

Previous rotating detectors usually set a large number of rotating anchors to obtain more accurate detection results for objects with arbitrary angles. In contrast, the anchor-selecting strategy of the SSD method is used by the proposed method, and only horizontal anchors instead of the oriented anchors are used so that the proposed method largely eliminates the influence of the angle and thus focuses more on shape matching. Furthermore, several times fewer anchors are required compared to methods based on oriented anchors, which greatly accelerates the training and interfacing process.

\subsection{Rotation-Decoupled Anchor Matching Strategy}

Based on the redefined bounding box, we implement a new rotation-decoupled anchor matching strategy. Before matching, the rotating bounding box/ground truth box is decoupled to a $\mathrm{HBB}_{\mathrm{h} / \mathrm{v}}$ and an acute angle, and the $\mathrm{HBB}_{\mathrm{h} / \mathrm{v}}$ is used as the new ground truth box for matching. Subsequently, the matching strategy similar to SSD [1] that is based on horizontal anchors is taken. Figure 3 shows the difference between the proposed strategy and the strategy based on oriented anchors. The IoU between the horizontal anchor and the decoupled ground truth box does not consider the angle, but the IoU between the rotating anchor and the ground truth box considers the angle. Specifically, anchors are assigned to ground truth boxes and considered as foreground (positive samples) when the IoU is greater than the given threshold; anchors are considered as background (negative samples) when the IoU is below another given threshold. In this study, the foreground IoU threshold is set to 0.5 and the background IoU threshold is set to 0.4 , as implemented in RetinaNet [2]. The proposed matching strategy suppresses the influence of angles and pays more attention to the matching of shapes. Thus, the ground truth boxes will naturally match the horizontal bounding boxes with the smallest angle to its principal axis, which avoids the periodicity of the angle.

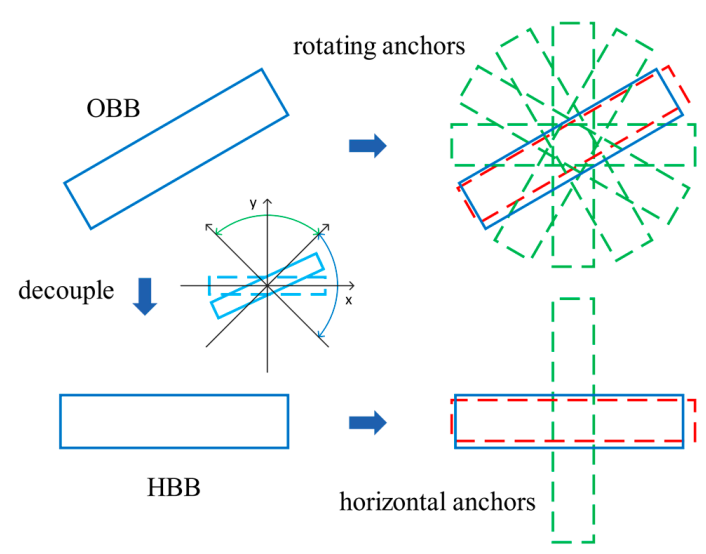

Figure 3. Rotation-decoupled matching strategy versus the strategy based on oriented anchors. The red bounding box indicates the matched anchor. 
For a better comparison, we simulate the matching process of the proposed strategy and the previous strategy based on oriented anchors, respectively. We use horizontal anchors at seven aspect ratios $\{1,2,1 / 2,4,1 / 4,8,1 / 8\}$. Anchors with three scales $\left\{2^{0}, 2^{1 / 3}, 2^{2 / 3}\right\}$ are subsequently added for denser scale coverage. Oriented anchors are obtained by adding a series of angles at $30^{\circ}$ intervals on horizontal anchors. Figure 4 shows an example of the matching results using the proposed strategy and the strategy based on oriented anchors. It can be seen that, despite the setting of dense anchors, the overlap between the ground truth box and the matched anchor is not high at some angles, which is due to the fact that the angles of the oriented anchors are set with fixed intervals without considering the aspect ratio, and anchors with a limited number of angles are subsequently used for matching. This problem is exacerbated by the sensitivity of the IoU to changes of angle. Further, we plot the change curves for the maximum IoU and the number of matched anchors under both strategies in Figure 5 under the condition of Figure 4. The foreground IoU threshold is set to 0.5. Figure 5 shows that for the strategy based on oriented anchors, the maximum IoU curve fluctuates sharply as the aspect increases, while the maximum IoU curve is unaffected by using the proposed strategy. The difference is more pronounced with the change of the matched anchor number. As the aspect increases, the difference in the number of matched anchors increases rapidly for oriented anchors with the same shape and different angles. In some cases, the number of anchors matching the ground truth box even reaches 0 , which means that the model will not be able to learn from the ground truth box. However, such a large difference is unreasonable. The ideal situation is that the matching result is not affected by the angle change. In contrast, the proposed strategy is always stable under various conditions.
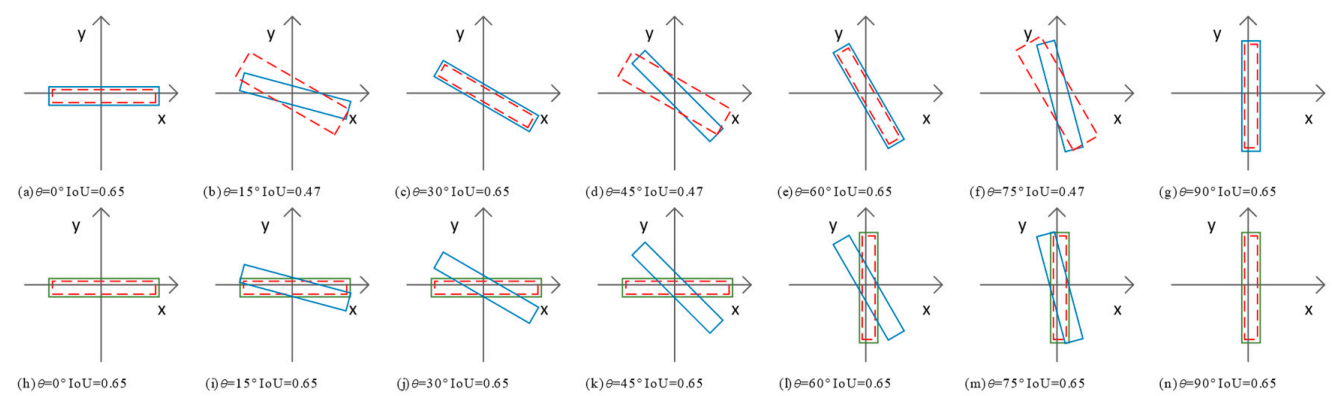

Figure 4. An example of matching results using the proposed strategy and OBB-based strategy. The top row demonstrates the matching results using the strategy based on oriented anchors and the bottom row presents the matching results using the proposed strategy. The blue box represents the ground truth box, the red box represents the anchor with the largest IoU matched and the green box represents the decoupled bounding box.
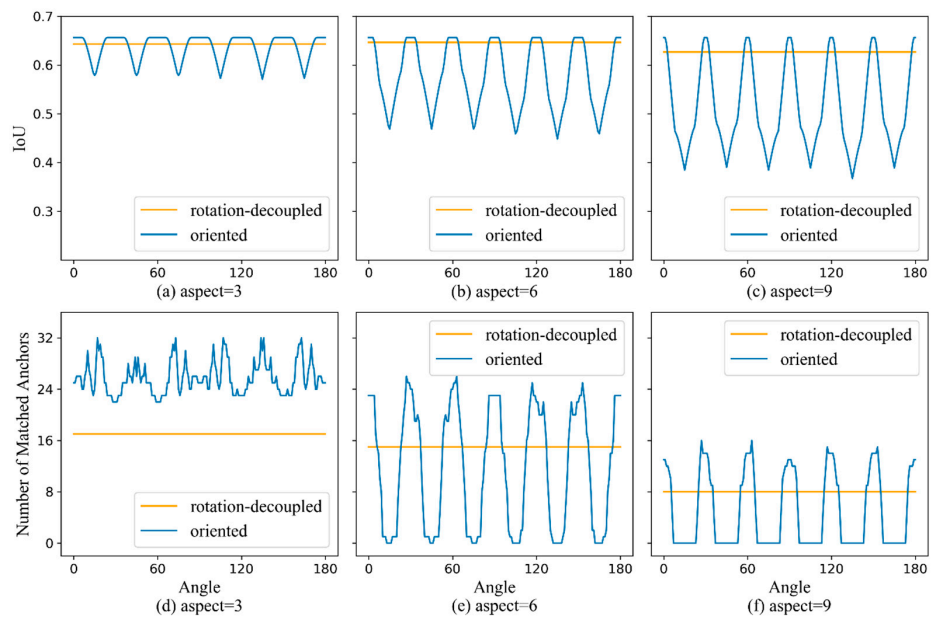

Figure 5. The comparison between the strategy based on oriented anchors and the proposed strategy. The top row shows the change in the maximum IoU with the angular change. The bottom row shows the change in the number of matched anchors with angular change. 


\subsection{Positive and Negative Sample Balance Strategy}

After the matching step, most anchors are labeled as background or as the negative class, while few are labeled as foreground or as the positive class. The foreground-background imbalance problem occurs during training and does not depend on the number of samples in each class [34]. We implement a balance strategy similar to Focal Loss [2], with the difference that the new strategy no longer dynamically scales the loss. We assign category labels of 1, 0 and -1 for foreground anchors and background anchors and ignored anchors. The corresponding binary cross-entropy loss is defined as follows:

$$
C E(y, p)= \begin{cases}-\log (p) & \text { if } y=1 \\ -\log (1-p) & \text { if } y=0 \\ 0 & \text { if } y=-1\end{cases}
$$

where $y$ is the class label of an anchor and $p$ is the predicted probability. Classification loss is defined as follows:

$$
L_{c l s}=\frac{1}{N_{p o s}} \sum_{i=1}^{N} C E\left(y_{i}, p_{i}\right)
$$

where $N$ indicates the number of anchors and $N_{\text {pos }}$ indicates the number of foreground anchors. Positive samples make a more stable contribution to classification loss than negative samples, but the number of positive samples is small compared to negative samples; $N_{\text {pos }}$ instead of $N$ is used for averaging, which can better solve the problem introduced by the sample unbalancing. Formulas (1) and (2) are key to the sample balancing strategy.

As with Faster R-CNN [24], the ground truth box $(x, y, w, h, \theta)$ and prediction box $\left(x^{*}, y^{*}, w^{*}, h^{*}, \theta^{*}\right)$ are encoded as $v=\left(t_{x}, t_{y}, t_{w}, t_{h}, t_{\theta}\right)$ and $v^{*}=\left(t_{x}^{*}, t_{y}^{*}, t_{w}^{*}, t_{h^{*}}^{*}, t_{\theta^{\prime}}^{*}\right)$ for position regression. The definition of $v$ and $v^{*}$ are listed in Equations (3) and (4). The anchors are expressed as $\left(x_{a}, y_{a}, w_{a}, h_{a}\right)$ :

$$
\begin{gathered}
t_{x}=\left(x-x_{a}\right) / w_{a}, t_{y}=\left(y-y_{a}\right) / h_{a} \\
t_{w}=\log \left(w / w_{a}\right), t_{h}=\log \left(h / h_{a}\right) \\
t_{\theta}=4 \theta / \pi \\
t_{x}^{*}=\left(x^{*}-x_{a}\right) / w_{a}, t_{y}^{*}=\left(y^{*}-y_{a}\right) / h_{a} \\
t_{w}^{*}=\log \left(w^{*} / w_{a}\right), t_{h}^{*}=\log \left(h^{*} / h_{a}\right) \\
t_{\theta}^{*}=\tanh \left(\theta^{*}\right)
\end{gathered}
$$

We adopt smooth-L1 loss for the rotation bounding box regression, and only the foreground anchors are included:

$$
\begin{gathered}
L_{\text {reg }}=\frac{1}{N_{\text {pos }}} \sum_{i=1}^{N_{p o s}} \operatorname{smooth}_{L 1}\left(v_{i}^{*}-v_{i}\right) \\
\operatorname{smooth}_{L 1}(x)= \begin{cases}0.5 x^{2} & \text { if }|x|<1 \\
|x|-0.5 & \text { otherwise }\end{cases}
\end{gathered}
$$

Finally, the multi-task loss is defined as

$$
L=L_{c l s}+\alpha L_{r e g}
$$

The trade-off between two terms is controlled by the balancing parameter $\alpha$. In the experiment presented in this paper, $\alpha$ is set to 1 .

\section{Experiments}

We evaluate the proposed detector on three public remote sensing datasets annotated with oriented bounding boxes, known as the DOTA [19], HRSC2016 [20] and UCAS-AOD [21] datasets. 


\subsection{Datasets and Settings}

DOTA is a large remote sensing dataset for object detection which contains 15 categories: plane (PL), baseball diamond (BD), bridge (BR), ground field track (GTF), small vehicle (SV), large vehicle $(\mathrm{LV})$, ship $(\mathrm{SH})$, tennis court (TC), basketball court (BC), storage tank (ST), soccer ball field (SBF), roundabout (RA), harbor (HA), swimming pool (SP) and helicopter (HC). DOTA contains 2806 aerial images collected from different sensors and platforms, including 1411 images for training, 937 images for testing and 458 images for validation. The size of each image is approximately $800 \times 800$ to $4000 \times 4000$ pixels. The dataset labels a total of 188,282 targets with both horizontal bounding boxes and oriented bounding boxes. We cropped the original image into sub-images of different sizes $\{512,768,1024,1536\}$ with an overlap of 0.25 and resize them to $768 \times 768$ for training and testing. We trained on the training set and validation set and evaluate the model on the test set. We train the proposed network for a total of 250,000 iterations, with an initial learning rate of 0.001 , which is then set to $1 \times 10^{-4}$ at 100,000 iterations and $2 \times 10^{-5}$ at 200,000 iterations.

The HRSC2016 dataset was built for the ship recognition task and collected 1061 images from Google Earth. The dataset contains 436 images including 1207 samples for training, 181 images including 541 samples for validation and 444 images including 1228 samples for testing. The image sizes range from $300 \times 300$ to $1500 \times 900$. We resized the images to $768 \times 768$ for training and testing. We trained on the training set and validation set and evaluated the model on the test set. We trained the proposed network for a total of 12,000 iterations, with an initial learning rate of 0.001 , which was then set to $1 \times 10^{-4}$ at 7500 iterations.

UCAS-AOD contains 1510 aerial images collected from Google Earth. Among them, 7482 planes are annotated in 1000 images and 7114 vehicles are annotated in another 510 images. These images have two sizes: $1280 \times 659$ pixels and $1714 \times 1176$ pixels. Since the dataset is not divided into a training set and test set, in [19,30,35], the authors randomly selected 1110 images for training and 400 for testing. Similar to these authors, we selected 400 images at regular intervals for testing, and the remaining 1110 images were used for training. We cropped the image into a series of sub-images whose length and width did not exceed 768 pixels. The model was trained by 30,000 iterations in total. The initial learning rate was set to 0.001 and changed from $1 \times 10^{-4}$ to $2 \times 10^{-5}$ at 15,000 iterations and 24,000 iterations, respectively.

We trained the model with a batch size of 12 on 1 Titan RTX GPU. The network was trained by an SGD optimizer, and the momentum and weight decay were set to 0.9 and $5 \times 10^{-4}$, respectively. The anchors had areas of $24^{2}$ to $384^{2}$ on pyramid levels P3 to P7. At each pyramid level, we used anchors at three scales $\left\{2^{0}, 2^{1 / 3}, 2^{2 / 3}\right\}$. We set different aspect ratios $\{1,2,1 / 2,4,1 / 4,8,1 / 8\},\{1.5,1 / 1.5,3$, $1 / 3,5,1 / 5,8,1 / 8\},\{1,2,1 / 2\}$ for DOTA, HRSC2016 and UCAS-AOD, respectively. In order to improve the robustness of the model, we used several data augmentation strategies, such as random photometric distortion [36], random horizontal, vertical flipping, random rotation, etc. Additional experiments with ResNet152 [33] as the backbone network kept the same settings except that the batch size was set to 6 . The code will be made public at https://github.com/Capino512/pytorch-rotation-decoupled-detector.

\subsection{Experimental Results}

Results on DOTA. We compare our results on DOTA with other state-of-the-art methods, as shown in Table 1 . The results are obtained by submitting the predictions to the official DOTA evaluation server. The existing detectors are mainly two-stage in DOTA dataset research, and their detection speed is usually slower than that of one-stage detectors. Benefiting from our designed rotation-decoupled anchor matching strategy, even compared to the most advanced two-stage detectors, the proposed single-stage detector achieves comparable performance while maintaining a simple network structure. Compared to various methods, our method achieves relatively stable detection results in all categories without any extra network design such as cascade refinement and an attention mechanism; furthermore, our method achieves the highest mAP, which is even higher than all other listed two-stage detectors. The effectiveness of the foreground-background class balance strategy also plays an important role. 
Table 1. Evaluation of the OBB task on the DOTA testing set. MS indicates that multi-scale images are used for testing. The abbreviations at the first line of this table can be referred to the introduction of DOTA at Section 4.1.

\begin{tabular}{|c|c|c|c|c|c|c|c|c|c|c|c|c|c|c|c|c|c|}
\hline Method & MS & PL & BD & BR & GTF & SV & LV & SH & TC & BC & ST & SBF & RA & HA & SP & HC & mAP \\
\hline \multicolumn{18}{|c|}{ Two-stage method } \\
\hline R-DFPN [37] & $x$ & 80.92 & 65.82 & 33.77 & 58.94 & 55.77 & 50.94 & 54.78 & 90.33 & 66.34 & 68.66 & 48.73 & 51.76 & 55.1 & 51.32 & 35.88 & 57.94 \\
\hline $\mathrm{R} 2 \mathrm{CNN}[38]$ & $x$ & 80.94 & 65.67 & 35.34 & 67.44 & 59.92 & 50.91 & 55.81 & 90.67 & 66.92 & 72.39 & 55.06 & 52.23 & 55.14 & 53.35 & 48.22 & 60.67 \\
\hline RoI-Transformer [13] & $\checkmark$ & 88.64 & 78.52 & 43.44 & 75.92 & 68.81 & 73.68 & 83.59 & 90.74 & 77.27 & 81.46 & 58.39 & 53.54 & 62.83 & 58.93 & 47.67 & 69.56 \\
\hline SCRDet [14] & $x$ & 89.41 & 78.83 & 50.02 & 65.59 & 69.96 & 57.63 & 72.26 & 90.73 & 81.41 & 84.39 & 52.76 & 63.62 & 62.01 & 67.62 & 61.16 & 69.83 \\
\hline SCRDet [14] & $\checkmark$ & 89.98 & 80.65 & 52.09 & 68.36 & 68.36 & 60.32 & 72.41 & 90.85 & 87.94 & 86.86 & 65.02 & 66.68 & 66.25 & 68.24 & 65.21 & 72.61 \\
\hline DRN+Hourglass-104 [17] & $x$ & 88.91 & 80.22 & 43.52 & 63.35 & 73.48 & 70.69 & 84.94 & 90.14 & 83.85 & 84.11 & 50.12 & 58.41 & 67.62 & 68.6 & 52.5 & 70.7 \\
\hline DRN+Hourglass-104 [17] & $\checkmark$ & 89.45 & 83.16 & 48.98 & 62.24 & 70.63 & 74.25 & 83.99 & 90.73 & 84.60 & 85.35 & 55.76 & 60.79 & 71.56 & 68.82 & 63.92 & 72.95 \\
\hline $\mathrm{R}^{3} \operatorname{Det}+\operatorname{ResNet101}[30]$ & $x$ & 89.54 & 81.99 & 48.46 & 62.52 & 70.48 & 74.29 & 77.54 & 90.80 & 81.39 & 83.54 & 61.97 & 59.82 & 65.44 & 67.46 & 60.05 & 71.69 \\
\hline $\mathrm{R}^{3}$ Det+ResNet152 [30] & $\hat{x}$ & 89.24 & 80.81 & 51.11 & 65.62 & 70.67 & 76.03 & 78.32 & 90.83 & 84.89 & 84.42 & 65.10 & 57.18 & 68.1 & 68.98 & 60.88 & 72.81 \\
\hline Ours+ResNet101 & $x$ & 89.70 & 84.33 & 46.35 & 68.62 & 73.89 & 73.19 & 86.92 & 90.41 & 86.46 & 84.30 & 64.22 & 64.95 & 73.55 & 72.59 & 73.31 & 75.52 \\
\hline Ours+ResNet101 & $\checkmark$ & 89.15 & 83.92 & 52.51 & 73.06 & 77.81 & 79.00 & 87.08 & 90.62 & 86.72 & 87.15 & 63.96 & 70.29 & 76.98 & 75.79 & 72.15 & 77.75 \\
\hline
\end{tabular}

Results on HRSC2016. The HRSC2016 dataset poses a huge challenge in terms the accuracy of the rotation detector since it contains a large number of ship instances with high aspect ratios and arbitrary orientation. Table 2 shows the comparison of the proposed model with other models. The times of the interface and post process are included when calculating the frames per second (FPS). The proposed method shows the effectiveness of detecting such targets. The proposed method learns the position information of the oriented object accurately without adding oriented anchors for angle regression. At the same time, benefiting from the simplicity of the implementation method, the proposed detector maintains a fairly high detection speed.

Table 2. Accuracy and speed comparison on HRSC2016. FPS: frames per second.

\begin{tabular}{ccccc}
\hline Method & Backbone & Input Size & mAP & FPS \\
\hline RRD [12] & VGG16 & $384 \times 384$ & 84.3 & - \\
RoI-Transformer [13] & ResNet101 & $512 \times 800$ & 86.2 & - \\
R $^{3}$ Det [30] & ResNet101 & $800 \times 800$ & 89.26 & 12 \\
R $^{3}$ Det [30] & ResNet152 & $800 \times 800$ & 89.33 & 10 \\
DRN [17] & Hourglass-104 & $768 \times 768$ & 92.7 & - \\
Ours & ResNet101 & $768 \times 768$ & 94.29 & 40 \\
Ours & ResNet152 & $768 \times 768$ & 94.61 & 31 \\
\hline
\end{tabular}

Results on UCAS-AOD. The UCAS-AOD dataset annotates two types of targets, airplanes and cars, which have relatively small sizes and cannot occupy the entire image. Therefore, only the feature maps on pyramid levels P3 to P6 are used. We train the model on the cropped image, and then make predictions on the uncropped original image. As shown in Table 3, the proposed detector also performs well on the UCAS-AOD.

Table 3. Accuracy comparison on UCAS-AOD. “Ours*” indicates that sResNet152 is used as the backbone.

\begin{tabular}{cccc}
\hline Method & Plane & Car & mAP \\
\hline DRBox [39] & 94.9 & 85 & 89.95 \\
S$^{2}$ ARN [40] & 97.6 & 92.2 & 94.9 \\
RetinaNet-H [30] & 97.34 & 93.6 & 95.47 \\
FADet [41] & 98.69 & 92.72 & 95.71 \\
R $^{3}$ Det [30] & 98.2 & 94.14 & 96.17 \\
Ours & 98.86 & 94.96 & 96.86 \\
Ours & 98.85 & 95.18 & 97.01 \\
\hline
\end{tabular}


Figure 6 provides a visual representation of our results on DOTA, HRSC2016 and UCAS-AOD datasets. It shows that our method has yielded fairly accurate detection results on each dataset.
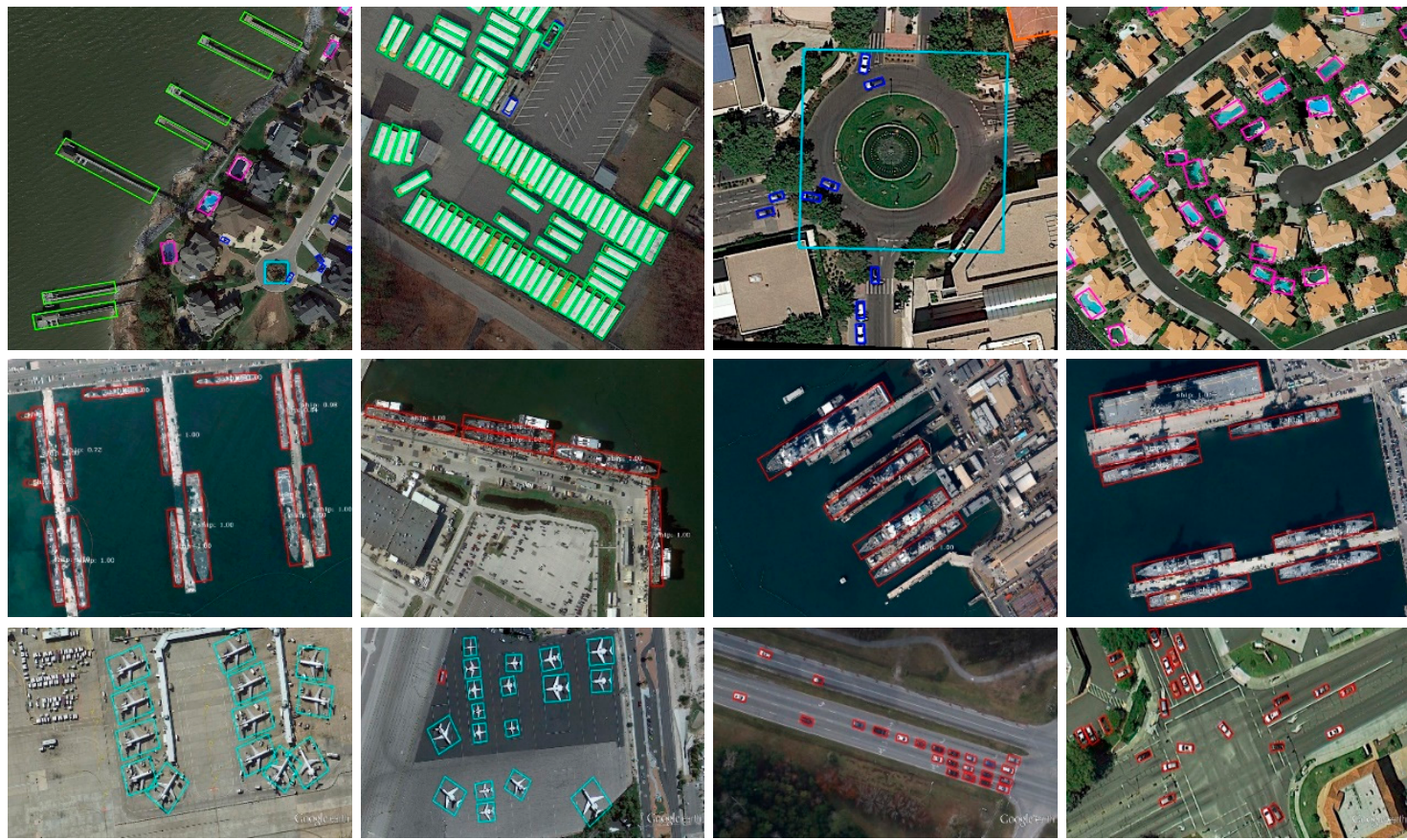

Figure 6. Example detection results of our method. The top row is from DOTA, the middle row is from HRSC2016 and the bottom row is from UCAS-AOD.

\section{Discussion}

The experimental results show that proposed method achieves desirable results on three different benchmarks without additional help. This demonstrates that our method is feasible and has good applicability. However, the proposed method also has the common problems of the current anchor-based method: (1) the anchors need to be set according to the shapes of the objects to be detected; (2) multi-scale anchors are used at each pyramid level, which will result in a large amount of calculation when the objects are of various shapes; and (3) it is difficult to detect highly overlapping objects. In the future, we will study how to reduce the dependence of the detector on multi-scale anchors and try to design a new single-stage detector to balance performance and efficiency.

\section{Conclusions}

In this study, we proposed a novel rotation-decoupled anchor matching strategy to simplify the anchor matching process for anchor-based methods. The new strategy optimizes the way in which the model learns the object position information without using rotating anchors. Instead of learning the shape and angle simultaneously, as in traditional rotating anchors, the proposed strategy learns the shape (using a horizontal anchor) and angle separately. Based on the proposed strategy, we build a single-stage rotation detector with a simple structure. The detector is accurate and efficient and can be further improved by adding advanced structures to it. The positive and negative sample balance strategy is applied to deal with the foreground-background imbalance problem encountered by single-stage detectors. We performed comparative experiments on multiple rotation detection datasets including DOTA, HRSC2016 and UCAS-AOD. The experimental results demonstrated that our method achieves state-of-the-art detection accuracy with high efficiency. 
Author Contributions: K.A. proposed the idea, developed the algorithm, conducted the experiments and wrote the paper. B.Z. gave many valuable suggestions and revised the paper. All authors have read and agreed to the published version of the manuscript.

Funding: This work was supported by the project titled "Research on integrated analytic algorithms using remote sensing big data and users' characteristic behaviors" under Grant Y920540K80.

Conflicts of Interest: The authors declare no conflict of interest.

\section{References}

1. Liu, W.; Anguelov, D.; Erhan, D.; Szegedy, C.; Fu, C.; Berg, A.C. SSD: Single Shot Multibox Detector. In Proceedings of the European Conference on Computer Vision, Amsterdam, The Netherlands, 8-16 October 2016; pp. 21-37.

2. Lin, T.-Y.; Goyal, P.; Girshick, R.; He, K.; Dollár, P. Focal loss for dense object detection. In Proceedings of the IEEE International Conference on Computer Vision, Venice, Italy, 22-29 October 2017; pp. 2980-2988.

3. Zhang, S.; Wen, L.; Bian, X.; Lei, Z.; Li, S.Z. Single-shot refinement neural network for object detection. In Proceedings of the 2018 IEEE Conference on Computer Vision and Pattern Recognition, Salt Lake City, UT, USA, 19-21 July 2018; pp. 4203-4212.

4. Tan, M.; Pang, R.; Le, Q.V. Efficientdet: Scalable and efficient object detection. In Proceedings of the IEEE/CVF Conference on Computer Vision and Pattern Recognition (CVPR), Nashville, TN, USA, 19-25 June 2020; pp. 10781-10790.

5. Lin, T.Y.; Maire, M.; Belongie, S.; Hays, J.; Perona, P.; Ramanan, D.; Dollar, P.; Zitnick, C.L. Microsoft coco: Common objects in context. In Proceedings of the 2014 European Conference on Computer Vision (ECCV), Zurich, Switzerland, 6-12 September 2014; pp. 740-755.

6. Everingham, M.; Van Gool, L.; Williams, C.K.; Winn, J.; Zisserman, A. The pascal visual object classes (voc) challenge. Int. J. Comput. Vision 2010, 88, 303-338. [CrossRef]

7. Liu, Z.; Hu, J.; Weng, L.; Yang, Y. Rotated region based CNN for ship detection. In Proceedings of the 24th IEEE International Conference on Image Processing (ICIP 2017), Beijing, China, 17-20 September 2017; pp. 900-904.

8. Liu, W.; Ma, L.; Chen, H. Arbitrary-oriented ship detection framework in optical remote-sensing images. IEEE Geosci. Remote Sens. Lett. 2018, 15, 937-941. [CrossRef]

9. Yang, X.; Sun, H.; Fu, K.; Yang, J.; Sun, X.; Yan, M.; Guo, Z. Automatic ship detection in remote sensing images from google earth of complex scenes based on multiscale rotation dense feature pyramid networks. Remote Sens. 2018, 10, 132. [CrossRef]

10. Tang, T.; Zhou, S.; Deng, Z.; Lei, L.; Zou, H. Arbitrary-oriented vehicle detection in aerial imagery with single convolutional neural networks. Remote Sens. 2017, 9, 1170. [CrossRef]

11. Li, Q.; Mou, L.; Xu, Q.; Zhang, Y.; Zhu, X.X. R ${ }^{3}$-Net: A deep network for multi-oriented vehicle detection in aerial images and videos. arXiv 2018, arXiv:1808.05560.

12. Liao, M.; Zhu, Z.; Shi, B.; Xia, G.S.; Bai, X. Rotation-Sensitive Regression for Oriented Scene Text Detection. In Proceedings of the IEEE Conference on Computer Vision and Pattern Recognition, San Francisco, CA, USA, 13-18 June 2018; pp. 5909-5918.

13. Ding, J.; Xue, N.; Long, Y.; Xia, G.S.; Lu, Q. Learning RoI Transformer for Detecting Oriented Objects in Aerial Images. In Proceedings of the IEEE Conference on Computer Vision and Pattern Recognition, Los Angeles, CA, USA, 16-19 June 2019.

14. Yang, X.; Yang, J.; Yan, J.; Zhang, Y.; Zhang, T.; Guo, Z.; Sun, X.; Fu, K. SCRDet: Towards More Robust Detection for Small, Cluttered and Rotated Objects. In Proceedings of the IEEE International Conference on Computer Vision, Seoul, Korea, 27 October-2 November 2019; pp. 8232-8241.

15. Li, C.; Xu, C.; Cui, Z.; Wang, D.; Jie, Z.; Zhang, T.; Yang, J. Learning Object-Wise Semantic Representation for Detection in Remote Sensing Imagery. In Proceedings of the IEEE Conference on Computer Vision and Pattern Recognition Workshops, Long Beach, CA, USA, 16-20 June 2019; pp. 20-27.

16. Xu, Y.; Fu, M.; Wang, Q.; Wang, Y.; Chen, K.; Xia, G.S.; Bai, X. Gliding vertex on the horizontal bounding box for multi-oriented object detection. IEEE Trans. Patt. Anal. Mach. Intell. 2020. [CrossRef] [PubMed] 
17. Pan, X.; Ren, Y.; Sheng, K.; Dong, W.; Yuan, H.; Guo, X.; Ma, C.; Xu, C. Dynamic Refinement Network for Oriented and Densely Packed Object Detection. In Proceedings of the IEEE/CVF Conference on Computer Vision and Pattern Recognition, Nashville, TN, USA, 19-25 June 2020; pp. 11207-11216.

18. Zhu, Y.; Du, J.; Wu, X. Adaptive period embedding for representing oriented objects in aerial images. IEEE Trans. Geosci. Remote Sens. 2020, 58, 7247-7257. [CrossRef]

19. Xia, G.S.; Bai, X.; Ding, J.; Zhu, Z.; Belongie, S.; Luo, J.; Datcu, M.; Pelillo, M.; Zhang, L. DOTA: A Large-Scale Dataset for Object Detection in Aerial Images. In Proceedings of the IEEE Conference on Computer Vision and Pattern Recognition, Salt Lake City, UT, USA, 18-23 June 2018; pp. 3974-3983.

20. Liu, Z.; Liu, Y.; Weng, L.; Yang, Y. A high resolution optical satellite image dataset for ship recognition and some new baselines. In Proceedings of the International Conference on Pattern Recognition Applications and Methods, Porto, Portugal, 24-26 February 2017; pp. 324-331.

21. Zhu, H.; Chen, X.; Dai, W.; Fu, K.; Ye, Q.; Jiao, J. Orientation robust object detection in aerial images using deep convolutional neural network. In Proceedings of the IEEE International Conference on Image Processing, Quebec City, QC, Canada, 27-30 September 2015; pp. 3735-3739.

22. Girshick, R.; Donahue, J.; Darrell, T.; Malik, J. Rich feature hierarchies for accurate object detection and semantic segmentation. In Proceedings of the IEEE Conference on Computer Vision and Pattern Recognition, Nashville, TN, USA, 19-25 June 2014; pp. 580-587.

23. Girshick, R. Fast R-CNN. In Proceedings of the IEEE International Conference on Computer Vision, Boston, MA, USA, 8-10 June 2015; pp. 1440-1448.

24. Ren, S.; He, K.; Girshick, R.; Sun, J. Faster r-cnn: Towards real-time object detection with region proposal networks. In Proceedings of the Advances in Neural Information Processing Systems, Montreal, QC, Canada, 7-12 December 2015; pp. 91-99.

25. He, K.; Gkioxari, G.; Dollar, P.; Girshick, R. Mask R-CNN. In Proceedings of the 16th IEEE International Conference on Computer Vision (ICCV 2017), Venice, Italy, 22-29 October 2017; pp. 2980-2988.

26. Redmon, J.; Divvala, S.; Girshick, R.; Farhadi, A. You only look once: Unified, real-time object detection. In Proceedings of the 2016 IEEE Conference on Computer Vision and Pattern Recognition, Las Vegas, NV, USA, 26 June-1 July 2016; pp. 779-788.

27. Law, H.; Deng, J. CornerNet: Detecting Objects as Paired Keypoints. In Proceedings of the European Conference on Computer Vision, Munich, Germany, 8-14 September 2018; pp. 765-781.

28. Zhou, X.; Wang, D.; Krähenbühl, P. Objects as points. arXiv 2019, arXiv:1904.07850.

29. Tian, Z.; Shen, C.; Chen, H.; He, T. Fcos: Fully convolutional one-stage object detection. In Proceedings of the IEEE International Conference on Computer Vision, Seoul, Korea, 27 October-2 November 2019; pp. 9627-9636.

30. Yang, X.; Liu, Q.; Yan, J.; Li, A.; Zhang, Z.; Yu, G. $\mathrm{R}^{3}$ det: Refined single-stage detector with feature refinement for rotating object. arXiv 2019, arXiv:1908.05612.

31. Yang, X.; Yan, J.; Yang, X.; Tang, J.; Liao, W.; He, T. SCRDet++: Detecting Small, Cluttered and Rotated Objects via Instance-Level Feature Denoising and Rotation Loss Smoothing. arXiv 2020, arXiv:2004.13316.

32. Lin, T.Y.; Dollár, P.; Girshick, R.; He, K.; Hariharan, B.; Belongie, S. Feature Pyramid Networks for Object Detection. In Proceedings of the IEEE Conference on Computer Vision and Pattern Recognition, Honolulu, HI, USA, 21-26 July 2017; pp. 936-944.

33. He, K.; Zhang, X.; Ren, S.; Sun, J. Deep residual learning for image recognition. In Proceedings of the 2016 IEEE Conference on Computer Vision and Pattern Recognition, Las Vegas, NV, USA, 27-30 June 2016; pp. 770-778.

34. Oksuz, K.; Cam, B.C.; Kalkan, S.; Akbas, E. Imbalance problems in object detection: A review. IEEE Trans. Pattern Anal. Mach. Intell. 2020. [CrossRef] [PubMed]

35. Azimi, S.M.; Vig, E.; Bahmanyar, R.; Körner, M.; Reinartz, P. Towards multi-class object detection in unconstrained remote sensing imagery. In Proceedings of the IEEE Asian Conference on Computer Vision, Perth, Australia, 4-6 December 2018; pp. 150-165.

36. Howard, A.G. Some improvements on deep convolutional neural network based image classification. arXiv 2013, arXiv:1312.5402.

37. Jiang, Y.; Zhu, X.; Wang, X.; Yang, S.; Li, W.; Wang, H.; Fu, P.; Luo, Z. R²CNN: Rotational Region CNN for Orientation Robust Scene Text Detection. arXiv 2017, arXiv:1706.09579. 
38. Jian, M.; Wei, S.; Hao, Y.; Li, W.; Hong, W.; Ying, Z.; Xiang, X. Arbitrary-oriented scene text detection via rotation proposals. IEEE Trans. Multimed. 2018, 20, 3111-3122.

39. Liu, L.; Pan, Z.; Lei, B. Learning a rotation invariant detector with rotatable bounding box. arXiv 2017, arXiv:1711.09405

40. Bao, S.; Zhong, X.; Zhu, R.; Zhang, X.; Li, M. Single Shot Anchor Refinement Network for Oriented Object Detection in Optical Remote Sensing Imagery. IEEE Access 2019, 99, 1. [CrossRef]

41. Li, C.; Xu, C.; Cui, Z.; Wang, D.; Zhang, T.; Yang, J. Feature-Attentioned Object Detection in Remote Sensing Imagery. In Proceedings of the IEEE International Conference on Image Processing, Taipei, Taiwan, 22-25 September 2019; pp. 3886-3890.

C 2020 by the authors. Licensee MDPI, Basel, Switzerland. This article is an open access article distributed under the terms and conditions of the Creative Commons Attribution (CC BY) license (http://creativecommons.org/licenses/by/4.0/). 